\title{
Study on the Significance and Value of Sports Activities and Sports Consumption from the Perspective of Semiotics
}

\author{
ZHANG Xueyan* \\ School of Media and Arts, Shanghai University of Sport, Shanghai, China
}

*Corresponding Authors: ZHANG Xueyan, School of Media and Arts, Shanghai University of Sport, Shanghai. China

\begin{abstract}
From the perspective of semiotics, this paper analyzes the symbol carrier, symbol code and symbol meaning of sports activities. The symbol carrier of sports activities can be the tangible sports items and sports goods, or the intangible sports culture idea. With the help of the characteristics of sports and the role of modern media, sports activities code symbols for sports activities. Sports activities embody the semiotic significance of competition, fitness, leisure and entertainment. At the same time, this paper probes into the symbol phenomenon and value of sports consumption, and analyzes the internal factors of the development of sports consumption market. The research shows that the symbolic value of sports consumption lies in the materiality and service of sports consumption, while sports consumption promotes the development of sports industry, there is also a certain lack of intrinsic value of sports. It is of great significance for the healthy and sustainable development of the sports industry to promote the harmonious and common development between sports activities and sports consumption and strengthen the positive guidance to the sports consumption market.
\end{abstract}

Key words: Semiotics, Sports activity, Sports consumption, Carrier, Coding

\section{INTRODUCTION}

Symbol is an important and special phenomenon in the development of human society. In ancient times, the totem of primitive man was one of the first symbols of human beings. In modern times, Saussure, a Swiss linguist, and Roland Barthes, a French Master of structuralism, made great contributions to the establishment and development of modern semiotics. In 1894, Saussure put forward the concept of semiotics. Saussure believed that language is a system of signs, and signs are composed of signifiers and signified. Roland Barthes, with the help of Saussure's linguistic theory, further revealed the operating mechanism of symbols in popular culture and decoded the popular culture in semiotics [1]. Roland Barthes made a breakthrough in Saussure's semiotics theory and successfully applied it to the study of popular culture. The practice shows that it is of great significance to study the social and cultural phenomena including sports from the unique research perspective of semiotics.

Sport is often regarded as a kind of symbol, which is a carrier with rich significance. Sport is closely related to honor and dignity, strength and success. It not only has great social potential, but also carries a lot of social and political significance. With the progress of science and technology and the rapid development of economy, the society is gradually dominated by consumption, and consumption is becoming more and more active, showing a development mode with social consumption as the core symbol value. People from the pursuit of survival needs, to the pursuit of social consumption value, consumption on the basis of their own development, gradually spread to all aspects of the society, become an important part of the ideological composition of modern consumer society. As the symbol elements of the integrated development of economy and culture, sports activities and sports consumption have developed rapidly in social life while the social consumption has gradually expanded.

\section{The Commercial Social Background Of Sports Semiotics}

With the rapid development of commodity economy, social commodities can be sold as symbols with a certain symbolic significance. In a society with highly developed economy and culture, the use value of commodities often exists after the cultural value of commodities. In the process of 
commodity consumption, consumers not only buy the use value of goods, but also buy the symbol value of goods. The more highly developed the commodity economy is, the more significant the symbolic characteristics of the commodity are.

Most of the commodities in today's society begin to move from seller's market to buyer's market. For producers, they must inject more meaning into their products in order to gain consumer recognition and survive in the fierce market competition. For consumers, when the use value of many commodities gradually tends to be similar, people begin to pay attention to the difference of commodities as symbols, and thus tend to pursue the meaning beyond the use value of commodities. Therefore, the commodity production in modern society is not comprehensive enough only with material production and marketing services. The commodity must also have cultural content and significance. By combining with cultural resources, the commodity can become a symbol of cultural significance that consumers are concerned about [2].

\section{The SEMiotic ANALYSIS OF SPORTS}

\subsection{The Symbol Carrier of Sports}

Sport, as a symbol carrier, is first manifested as various specific sports activities. According to the theory of Roland Barthes, whether running, jumping, throwing and other track and field events, as well as basketball, football, volleyball and other ball events, or mountaineering, diving, aerobics and other sports activities, these are an important part of sports symbols. Sports as a social cultural phenomenon in the economic backdrop, there exists a social stratification in the field of sports, sports is with class logo, for example: in the upper social class choices such as golf, middle class choose the tennis and so on, and the medium is the class to choose more football, basketball and so on. The sports consumption level of the participants is different, and the sports they participate in are also different. Therefore, according to the sports people often participate in, it is easier to identify the social class attributes of sports participants.

Golf, tennis, football and other sports are the symbol carriers of sports. As the carrier of symbols, they are endowed with certain social significance and can be recognized by the public under specific social conditions. As a symbol carrier, sports can be both tangible sports goods and intangible culture and ideas, such as sports brand and sports monopoly culture. The expensive jerseys, scarves, casual clothes and accessories in the exclusive stores of famous football clubs can all be regarded as a symbol carrier of sports.

\subsection{Symbolic Coding of Sports}

According to the characteristics of sports, sports activities and sports consumption are coded with symbols. First of all, sports usually aim at competition. Sports pursue strength, speed and difficulty. Therefore, sports are generally associated with the strong, so that sports, according to their own characteristics, carry on the symbol coding for sports activities. Secondly, "life lies in movement", sports is the main feature of physical movement, so there is a close connection between sports and health. In people's common sense, often engaged in physical activities and have excellent performance in physical activities, become an important standard to judge people's health.

With the continuous progress of society, people participate in sports activities, not only for physical fitness, but also for a kind of value identification, the pursuit of spiritual relaxation and sentiment edification. Modern media rely on the powerful communication ability, affecting people's values, through the continuous communication of the media, people know the most can reflect the value and taste of sports activities. For a long time, sports activities have been divided into different items so as to provide choices for people with different needs. Modern media has also completed the symbol coding of sports.

\subsection{Symbolic Significance of Sports}

(1) Sport is the symbol of competition. Since the goal of sports competition is the only one, athletes in order to achieve success must do their best to overcome difficulties, overcome their opponents, and overcome themselves. Competitiveness in sports has much in common with hard work and struggle in the course of life. At the same time, the competition of sports is very similar to the fierce market competition. Sports fully explain the meaning from life to market competition, therefore, sports in the 
contemporary society as a symbol of competition and can be popular. For individuals, sports have a characteristic of transcending oneself. Sports can sharpen one's will and enhance one's sense of competition. For companies or enterprises, inviting sports champions as product spokesmen also takes advantage of the sports symbol meaning that champions are strong to shape the image of strong, so as to attract more consumers to choose their products [3].

(2) Sport is the symbol of health. Modern society has a high pace of life, highly developed transportation facilities and high nutrition of dietary products. This brings people efficiency and comfort at the same time, but also makes people's physical quality invisible increasingly weakened. In order to avoid the occurrence of modern civilization disease, people increase health investment and take an active part in physical exercise. For people, physical activities can stimulate and regulate people's muscles and nerves well, so that people feel happy and full of strength. What's more, as a symbol of health, physical education is conducive to strengthening physical fitness and good for health. With the highly developed modern media, the idea that "Sports is a healthy way of life" has been deeply rooted in people's minds. Therefore, nowadays sports activities have become a psychological demand to realize people's pursuit of high quality life and health.

(3) Sports is the symbol of communication and recreation. Sports can effectively promote the communication and interaction between people. For example, when greeting strangers on the court, people will not feel embarrassed, but will feel friendly and natural. In real life, many of the interactions and friendships between people begin with shared sports. In addition, sport is both a competition and a game. People need to follow the common rules, through their own efforts, overcome difficulties, in order to achieve the set goals. The playability of sports provides people with opportunities and possibilities for relaxation and recreation. With the improvement of people's living standard, the consumption type begins to change from material consumption to service consumption, and sports consumption industry appears. In the context of the rapid development of sports industry, leisure sports have been recognized by people for their characteristics of interest, low utility and weak competitiveness. Sport has become a means for people to enjoy leisure and entertainment and to pursue self-relaxation.

\section{THE SYMBOLIZATION OF SPORTS CONSUMPTION PHENOMENON IN MODERN SOCIETY}

Nowadays, with the rapid development of social economy, people often spend their leisure time on a series of social consumption activities, including leisure sports activities. People's demand and consumption consciousness become important factors, and become the main driving force of the market economy, thus stimulating the growth of consumption. In social consumption, material products enter the social consumption market and begin to form their own social value attributes. People's consumption activities are no longer simply the feelings of the consumption process, but reflect the intrinsic value of people through consumption activities. The substance consumed by people is no longer limited to the material, but reflects the cultural value attached to consumption through consumption, showing the comprehensive associated value of consumption, namely the socalled symbolic value of consumption [4].

Symbol has been integrated into all aspects of social consumption, and gradually become people's internal consumption values. For example, the popular social consumption of famous sports brands such as Nike, YONEX, LI NING, and ASICS, the symbolic value of consumption has a particularly significant performance in the current society. People pay more attention to symbolic consumption, and consumption changes from use value to symbol value, and the demand for use value of consumer goods turns to the pursuit of consumption value, thus making social value gradually turn to symbolization [5].

\section{SPORTS CONSUMPTION In SYMBOL CONSUMPTION ENVIRONMENT}

\subsection{Value of Sports Consumption in Consumer Society}

Modern sports consumption can be divided into two types, namely: physical sports consumption and non-physical sports consumption. The concept of physical sports consumption is defined by the physical goods related to sports, including: sports equipment, supplies, sports books, newspapers and other physical products. Non-physical sports consumption can be divided into two categories, namely, 
spectator consumption and participatory consumption. The concept of non-physical sports consumption refers to the related products attached to sports, including sports performance industry and participatory consumption.

From the perspective of the environment of sports consumption, the external environment and internal value of society promote the rapid development of sports consumption, and become the main driving force for the progress of sports consumption. People are getting richer and have the ability to strengthen sports consumption. In people's mind, the idea of "health first" tends to dominate, thus making sports consumption become an important part of the consumption society [6].

\subsection{The Symbolization of Physical Sports Consumption}

Materiality sports consumption has been ubiquitous in the consumption society, while service sports consumption is still in the development stage at present. In the consumption society with rapid economic development, material life has been greatly improved, and people use part of their income for their own consumption needs. In terms of service sports consumption, the previous physical sports consumption demand has gradually turned to the symbolization of sports consumption. For example, in the purchase of sports equipment and sports goods, most buyers have little understanding of the difference in the use value of sports goods, and sports consumer behavior sometimes has a certain "blind obedience". At this time, the sports brand often becomes the value judgment standard of consumers. International famous sports goods brands often become the target of people's consumption and purchase. At the moment, the value of the brand surpasses the inherent value of sports goods and becomes the primary factor for people to consider buying sports goods, which reflects people's pursuit of the symbolization of sports consumption goods [7].

\subsection{The Symbolization of Non-Physical Service Sports Consumption}

In various sports items and industries, the consumption symbolization of service sports consumption is also reflected. For example, in the sports competition performance in the sports service industry, the value of symbols is to meet the needs of consumers through watching the performance. From the development of sports competition consumption market, all kinds of commercial competitions have gradually become the focus of sports competition consumption market. For example: the NBA, the French Open, FIFA World Cup and so on. The sports commercial performance has strong symbolic characteristics. The value of symbols lies in meeting the inner needs of consumers, and the consumption purpose is gradually transformed into the inherent symbolic value of sports events. When the symbol value is dominated by the consumer social market, the commercial value and profit of sports events become the purpose of holding the events, which promotes the rapid development of sports competitions and sports consumption market [8].

More and more people are participating in all kinds of sports, including some high-grade sports. For example: Tennis, golf, snooker and so on. Under the guidance of the symbolization of sports consumption, the sports consumption market shows the symbolic value outside of sports [9]. For example, in golf, the number of participants is small, but the level of attention is high. Because to some extent, the golf game reflects the social status of the participants, People pay more attention to the social value of aristocratic sports attributes brought by sports consumption itself, rather than just the use value of sports consumption. This is the fundamental change of a symbol of sports consumption demand. Sports consumption demand is no longer only to strengthen physical exercise and shape beauty, but to reflect the social value connotation of sports items. Therefore, sports consumption eventually becomes symbol consumption and value pursuit [10].

\section{VALUE DEVELOPMENT OF SPORTS CONSUMPTION SyMbOLIZATION}

\subsection{Strengthen the Industrial Development of Sports Consumption Structure}

In the sports consumption market, the transformation of sports consumption symbol and value can promote the development of the whole sports industry. The structure of sports industry will change with the change of sports consumption structure and consumption demand, and the demand of sports consumption creates a huge market demand for the development of sports industry. With the rapid development of the value of sports symbols, the sports industry should set up a marketing concept to deal with the sports consumption symbol market. Sporting goods industry should also gradually to develop strategic focus, go to the shape of our sports goods brand and marketing, enterprises can 
undertake and sponsor some big sports events to show their own brand goods, so as to improve the visibility of the brand, fully embodies its symbolic value, and get more attention to group [11]. The rapid growth of sports consumption market has brought unprecedented development to the sports service industry, and the changing trend of sports consumption demand and market trend will have an important role in promoting and promoting the sports industry.

\subsection{Guide the Development Direction of Sports Value in the Symbolization of Sports Consumption}

The harmonious development of the sports consumption market and the sports industry is an important guarantee for the sustainable and healthy development of the sports industry. However, with the rapid development of the sports industry, the modern sports industry will deviate from the sports consumption that people pay attention to, especially the inherent nature of sports may be more and more far away. In the consumption environment of modern society, people develop sports resources for the purpose of obtaining more benefits. Therefore, the sports industry needs to change its previous development mode and develop sports products suitable for the public consumption concept according to the symbolic value of sports resources [12].

In the process of the development and expansion of the sports consumption market, the brand awareness of sports consumption has attracted much attention. Events like the NBA basketball tournament, the French Open, and the soccer World Cup are getting bigger and bigger. Obviously, the prosperity of sports consumption is of great help to promote the sports industry, but it leads to the weakening of the intrinsic value of sports, and sports consumption gradually turns into the pursuit of famous brands, stars and high ratings. The sports industry under the symbol development mode, the development mode should present multi-polarization, people should turn their attention to sports to the national fitness and the development of the sports industry, pay attention to and organize largescale sports events, and guide people to actively participate in the national sports fitness, which is the key to the development of the sports industry.

\subsection{Integration of the Awakening of Sports Value and the Development of Sports Industry}

The sports industry needs people's extensive attention and participation, so that it can survive and develop in the economic environment, and the symbolic value of sports participation can be highlighted. Therefore, coordinating the relationship between the sports industry and sports consumption symbols, to solve the problems of developing sports industry and sports consumption, sports participation guide people to the sports industry, promote the enthusiasm of the masses to participate in sports activities with enthusiasm, and expand the scale of sports consumption market, the sports industry to achieve continuous healthy development. This is an important development direction for the harmonious development of sports industry and sports consumption market.

\section{CONCLuSiON}

At present, through modern media technology, sports and all aspects of society are more and more closely connected, sports quickly into the public life, and become a popular way of entertainment. In this case, sports is brought into the mass culture operation, sports is abstracted as a symbol, sports activities and sports consumption carry more rich social significance.

The symbol carrier of sports activities can be tangible sports items and sporting goods, or intangible cultural ideas. Sports influence people's values, and with the help of its own characteristics and modern media, sports activities for the symbol code. Physical activity embodies the symbolic significance of competition, health, communication and recreation.

The connotation of the symbolic value of sports consumption lies in the materiality and service of sports consumption. While sports consumption promotes the development of sports industry, it also leads to the lack of intrinsic value of sports. It is necessary to promote the harmonious coordination and common development between sports activities and sports consumption so that the sports consumption market and sports industry can develop healthily and sustainably.

\section{REFERENCES}

[1] WANG Shumei. Consumer society and people's life symbolic, The Northern Review, 2007 (2): 137-140 
Study on the Significance and Value of Sports Activities and Sports Consumption from the Perspective of Semiotics

[2] ZHOU Ersan. Semiotics of contemporary sports interpretation, Journal of TIANJIN Sports College, 2006.21 (6): 547-548

[3] YANG Yun. Symbolization of sports consumption and its value analysis, Journal of NANJING Institute of Sport, 2011.25 (3): 13-16

[4] ZHANG Jianhui, ZHONG Bing-shu. Golf's symbol consumption, Journal of Sport, 2009 (9): 36-38

[5] QIU Jingxin. Consumer culture: the key points in promoting sports product brand, Journal of GUANGZHOU Sports Institute, 2005 (4): 56-58

[6] ZHAO Ling. Consumption of the humanistic connotation and its value regression, Philosophy Research, 2006 (9): 111-114

[7] HAN Jiao. Sports consumption and the inner mechanism of the interaction between sports industry and countermeasures study, Journal of Economic Study Tribune, 2017.32:46-47

[8] ZHANG Yongjun. On sports consumption from the perspective of symbol consumption, Journal of CHENGDU University of Physical Education, 2006(1):13-16.

[9] XU Duowei. Macro cognition and understanding of sports consumption, Journal of HUBEI University of Economics, 2017.14(7):37-39.

[10] WU Feng. Analysis on the promoting value of national fitness campaign to the development of sports industry in China, Sports and Science, 2010(4):69-72.

[11] ZHANG Lin, CHEN Xiyao, ZHONG Tianlang. The development conception and prospect of China's sports industry in the next 5 years, Sports Science, 2006 (7):13-19.

[12] ZHANG Ke. Research on the status quo and development strategy of sports consumption industry in digital media era, New Media Research, 2021(1):89-92.

\section{AUTHOR'S BIOGRAPHY}

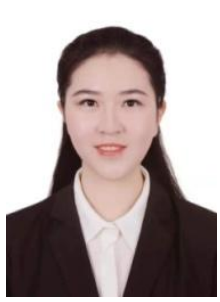

ZHANG Xueyan, Master of Fine Arts (MFA), School of Media and Arts, Shanghai University of Sport, Shanghai, China. The main research direction is research on modern media art. Email: dchzhang2007@163.com

Citation: ZHANG Xueyan. "Study on the Significance and Value of Sports Activities and Sports Consumption from the Perspective of Semiotics" International Journal of Managerial Studies and Research (IJMSR), vol 9, no. 8, 2021, pp. 19-21. doi: https://doi.org/10.20431/2349-0349.0908003.

Copyright: () 2021 Authors. This is an open-access article distributed under the terms of the Creative Commons Attribution License, which permits unrestricted use, distribution, and reproduction in any medium, provided the original author and source are credited. 